\title{
Classes of critical avalanche dynamics in complex networks
}

\author{
Filippo Radicchi, ${ }^{1, *}$ Claudio Castellano $\odot,{ }^{2}$ Alessandro Flammini, ${ }^{1}$ Miguel A. Muñoz $\odot,{ }^{3}$ and Daniele Notarmuzi ${ }^{1}$ \\ ${ }^{1}$ Center for Complex Networks and Systems Research, Luddy School of Informatics, Computing, and Engineering, Indiana University, \\ Bloomington, Indiana 47408, USA \\ ${ }^{2}$ Istituto dei Sistemi Complessi (ISC-CNR), Via dei Taurini 19, I-00185 Roma, Italy \\ ${ }^{3}$ Departamento de Electromagnetismo y Física de la Materia e Instituto Carlos I de Física Teórica y Computacional Facultad de Ciencias \\ Universidad de Granada E-18071, Granada, Spain
}

(Received 10 December 2019; accepted 7 July 2020; published 30 July 2020)

\begin{abstract}
Dynamical processes exhibiting absorbing states are essential in the modeling of a large variety of situations from material science to epidemiology and social sciences. Such processes exhibit the possibility of avalanching behavior upon slow driving. Here, we study the distribution of sizes and durations of avalanches for well-known dynamical processes on complex networks. We find that all analyzed models display similar critical behavior, characterized by the presence of two distinct regimes. At small scales, sizes, and durations of avalanches exhibit distributions that are dependent on the network topology and the model dynamics. At asymptotically large scales instead-irrespective of the type of dynamics and of the topology of the underlying network—sizes and durations of avalanches are characterized by power-law distributions with the exponents of the standard mean-field critical branching process.
\end{abstract}

DOI: 10.1103/PhysRevResearch.2.033171

\section{INTRODUCTION}

In this paper, we study seven stochastic models that are prototypical to describe the diffusion of some sort of "activity" in networks [1,2]. Specifically, our analysis includes: the competition-induced-criticality model (CIC) [3-5], the voter model (VOT) [6,7], the invasion process (IP) [8], link dynamics (LD) [9], the contact process (CP) [10], the susceptibleinfected-susceptible model (SIS) and the susceptible-infectedrecovered model (SIR) [2] (see Fig. 1). In all these models, active nodes can pass the active status to their inactive neighbors in the network, and can return to the inactive status either spontaneously or by interacting with inactive neighbors. The rules that govern these transitions are model specific. All of the models are characterized by the existence of one or more absorbing states, at which all dynamics ceases and the system state remains frozen. Typically, when parameters are set such that the system is at the interface between the active and inactive/absorbing phases, critical behavior-characterized by power-law distributions of the sizes and durations of activity avalanches-emerges.

Many empirical studies of spreading phenomena in social, technological and biological networks [11-13] reveal such a critical behavior. These observations have triggered interest in understanding the origin of criticality in specific dynamical models and its relationship with the underlying network

\footnotetext{
*filiradi@indiana.edu

Published by the American Physical Society under the terms of the Creative Commons Attribution 4.0 International license. Further distribution of this work must maintain attribution to the author(s) and the published article's title, journal citation, and DOI.
}

architecture. The existing literature reveals that the statistical properties of avalanches in some of the above models may be dependent on the topology of the network on top of which the dynamics proceeds $[4,14-16]$, while some other authors suggest that critical features are independent of network topology [17]. Here, we aim at reconsidering the problem of critical scaling behavior on networks, for all the above dynamical models within a common and coherent perspective.

In many cases, the dependence of avalanche statistics on the topology of the underlying network is theoretically explained by regarding the avalanche as the result of a simple branching process (BP) [18-21], where the network outdegree distribution $P\left(k^{\text {out }}\right)$ is identified with the distribution of offspring number. Such a mapping is exact as long as the evolution of an avalanche does not substantially change the probability for an active node to find inactive neighbors to infect, e.g., when the substrate is a directed tree. However, the mapping usually fails for arbitrary networks: after some transient time an active site may find neighbors that are already active, so that the process is not merely branching out, but interfering with itself. Reasonably, this failure is more dramatic in undirected networks as the front of an avalanche can immediately move backwards and, as a consequence, break the equivalence with a simple branching process.

According to the standard BP theory, sizes $S$ and durations $T$ of avalanches at criticality-i.e., when on average there is one offspring per active node-are distributed according to power laws

$$
P(S) \sim S^{-\tau} \mathcal{G}_{\mathcal{S}}\left(S / S_{C}\right) \text { and } P(T) \sim T^{-\alpha} \mathcal{G}_{\mathcal{T}}\left(T / T_{C}\right),
$$

where $\tau$ and $\alpha$ are avalanche critical exponents, and $\mathcal{G}_{S}\left(S / S_{C}\right)$ and $\mathcal{G}_{\mathcal{T}}\left(T / T_{C}\right)$ are cut-off (scaling) functions, with the cutoff scales, $S_{C}$ and $T_{C}$, depending only on system/network size right at the critical point $[22,23]$. Moreover, the average 


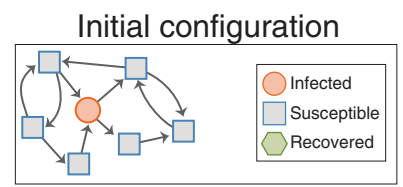

Contact process

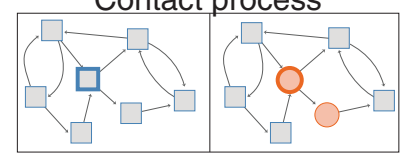

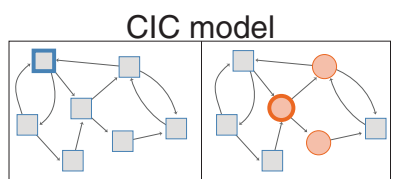

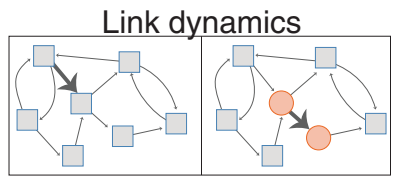

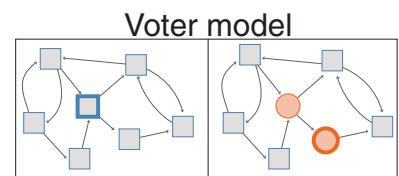

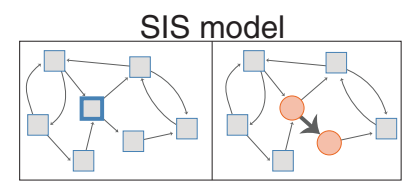

Invasion process
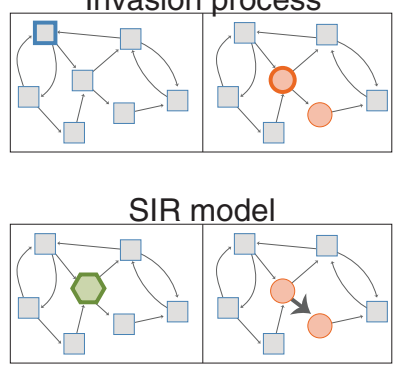

FIG. 1. Models for avalanche dynamics on networks. The figure serves as a schematic illustration to emphasize differences and similarities between the various dynamical models considered in this paper. The upper-left panel depicts an initial configuration, where a single node is in the "active" state, whereas all other nodes are inactive. The rest of the panels display configurations reachable after one elementary reaction. Depending on the model, elementary reactions are triggered by randomly selected nodes, edges, or both. The elements triggering the elementary reactions are highlighted in the various panels. For clarity of the illustration, we report for each model only two of the possible configurations that can be reached after one elementary reaction. Specifically, configurations appearing in the left panels are reached after a recovery reaction, whereas configurations appearing in the right panels are obtained after a spreading reaction (in both cases the nodes or links triggering the reaction are highlighted with thick lines). Detailed definitions of all dynamical models can be found in Ref. [26].

avalanche size scales with its duration as $\langle S\rangle \sim T^{\theta}$, where the exponent $\theta$ obeys the general scaling relationship $\theta=$ $(\alpha-1) /(\tau-1)[24,25]$.

The values of $\tau$ and $\alpha$ may depend on the offspring distribution, i.e., the probability for an active node to activate a given number of new nodes. If the second moment of such a distribution is finite, then

$$
\tau=3 / 2, \quad \alpha=2 \text { and } \theta=2 .
$$

Equation (2) defines the so-called "standard" mean-field (MF) or "branching process" exponents. Thus, this type of scaling is expected to emerge for critical avalanches in the case in which the second moment of $P\left(k^{\text {out }}\right)$ in the network is finite.

In fact, these values of $\tau$ and $\alpha$ are extremely universal and robust; they emerge in many different types of propagation processes such as directed percolation, CP, VOT, SIS, SIR, and many others, as long as the underlying pattern of connections is either a high-dimensional lattice or a sufficiently homogeneous network [23,27-30]. ${ }^{1}$ This superuniversality can be rationalized using a Langevin equation for the density $\rho$ of active sites

$$
\frac{d \rho(t)}{d t}=F \sqrt{\rho(t)} \xi(t)
$$

where $F$ is a noise-amplitude constant and $\xi(t)$ a zero-mean Gaussian white noise, which is shared, as an effective meanfield description, by all the above mentioned models [31]. Observe that the square-root term in Eq. (3) accounts for "demographic" fluctuations and is a direct consequence of the central limit theorem $[30,35]$.

On the other hand, if the second moment of the offspring distribution-or, equivalently $P\left(k^{\text {out }}\right)$-diverges, then the

\footnotetext{
${ }^{1}$ A particularly simple proof of the emergence of the standard exponents when the underlying tree is homogeneous with $k^{\text {out }}=$ 2 can be found in Ref. [31]. A more systematic derivation-for different types of underlying regular or random tree topologies-can be obtained within the generating-function formalism [29,32,33]; for instance, already back in 1949, Otter computed the solution when $P\left(k^{\text {out }}\right)$ is a Poisson distribution [34].
}

critical exponents of the associated branching process differ from the standard MF ones of Eq. (2). In particular, for $P\left(k^{\text {out }}\right)$ $\sim\left[k^{\text {out }}\right]^{-\gamma}$ with $2<\gamma<3$, one obtains $\gamma$-dependent exponents $[4,36,37]$,

$$
\tau=\frac{\gamma}{\gamma-1}, \quad \alpha=\frac{\gamma-1}{\gamma-2}, \quad \text { and } \theta=\frac{\gamma-1}{\gamma-2} .
$$

Observe that these "anomalous" branching process (ABP) exponents converge to those of Eq. (2) in the limit $\gamma \rightarrow 3$, i.e., when the second moment of $P\left(k^{\text {out }}\right)$ becomes finite (with the caveat of logarithmic corrections to scaling at the marginal value $\gamma=3[36])^{2}$

Real-world networks often exhibit power-law degree distributions $P(k) \sim k^{-\gamma}$ with $2<\gamma<3$ [39,40], and studies concerning spreading processes often assume underlying scalefree network topologies [1,2]. A naive extension of the standard BP mapping to networks with diverging second moment of the degree distribution suggests that one should generically observe anomalous exponents. Does anomalous scaling hold for avalanches on real networks?

The current literature on the existence of anomalous avalanche scaling in scale-free networks reports conclusions that are often contradictory or difficult to reconcile with each other. For example, according to Larremore et al. critical avalanches on networks are always characterized by the standard MF exponents of Eq. (2) irrespective of the underlying network topology [17]. However, numerical evidence in support of such a claim is presented only for power-law networks with degree exponent $\gamma>3$. Furthermore, this is in apparent contradiction with what reported for the CIC model

\footnotetext{
${ }^{2}$ Actually, the above-mentioned effective Langevin-equation approach breaks down for $\gamma<3$, as it includes a standard Gaussian noise, stemming ultimately from the central limit theorem for the addition of stochastic variables with finite variance. In the case $\gamma<3$, the variance of the variables to be added is not finite, the Gaussian noise needs to be replaced by a Lévy-stable distribution, leading to a different type of effective description and to anomalous behavior [i.e., Eq. (4)] [38].
} 
on directed scale-free networks. In particular, Gleeson et al. employ a map onto an anomalous branching process to argue that one should expect anomalous scaling for $2<\gamma<3$ and standard MF critical exponents for $\gamma>3$ [4], but offered limited computational evidence in support of such a claim.

Also for the broadly studied SIR model the current state of understanding is not entirely clear. First, the model is never studied directly; rather, claims follow from SIR equivalence with bond percolation [41], according to which the distribution of SIR avalanche sizes can be deduced from the percolation cluster-size distribution. Theoretical claims on bond percolation in scale-free networks mostly regard undirected networks [14-16]. This is a very difficult setting to consider given that the percolation threshold vanishes [42]. A large-scale numerical study of the percolation cluster size distribution in scale-free graphs is the one of Ref. [43], where critical exponents seem compatible with the standard ones of Eq. (2) for any $\gamma>2$.

The goal of this paper is to provide a coherent picture for avalanche statistics in critical processes taking place on networks. The study consists in extensive numerical simulations, combined with analytical arguments, of the various avalanche models on a variety of networks, both synthetic and real.

\section{MODELS: NETWORKS AND DYNAMICS}

The models studied here are described in detail in Ref. [26]. Figure 1 illustrates schematically the mechanisms at the basis of the various models under consideration. As a substrate for the dynamics of activity, we assume in all cases a network composed of $N$ nodes. The topology of the network is fully specified by its adjacency matrix $A$, whose generic element $A_{i j}=1$ if an edge from node $i$ to node $j$ exists and $A_{i j}=$ 0 otherwise. We assume that no self-loops are present in the network, i.e., $A_{i i}=0$ for all $i$. In the most general case, we consider directed networks, where $A_{i j} \neq A_{j i}$. For simplicity, we further assume that the network is composed of only one strongly connected component, so that at least one directed path between any pair of nodes exists.

The state of the system at time $t$ is denoted by the vector $\vec{\sigma}(t)=\left[\sigma_{1}(t), \sigma_{2}(t), \ldots, \sigma_{N}(t)\right]^{T}$, where $\sigma_{i}(t)$ is a discretevalued variable representing the state of node $i$ at time $t$. In all models except for the SIR, $\sigma_{i}(t)$ can assume two values: $\sigma_{i}(t)=1,0$ indicating that the node is active, inactive respectively. In the SIR one can also have that $\sigma_{i}(t) \neq 0,1$ meaning that node $i$ is recovered and does not participate any longer in the dynamics.

All models are stochastic Markov processes where the elementary reactions that lead to changes in system configurations are triggered by the random selection of network elements, either nodes, edges or both. Propensities of the various reactions may depend on exogenous parameters whose values can be tuned to bring the system into different dynamical regimes. All models are characterized by an asynchronous updating scheme, meaning that an elementary dynamical step leads to the change of the state of at most all neighbors of a single node. The state $\vec{\sigma}=\overrightarrow{0}=(0,0, \ldots, 0)^{T}$ is an absorbing configuration for all models. Additional absorbing configurations are present in some models. For example, in the SIR model, all configurations with no infected nodes, but arbitrary number of recovered nodes are absorbing; in some other models, such as the CIC, the configuration $\vec{\sigma}=\overrightarrow{1}=$ $(1,1, \ldots, 1)^{T}$ is also absorbing at the critical point.

We are interested in the critical regimes of the considered dynamical models. The criterion to achieve criticality is model specific. VOT, IP, and LD have no free parameters and are intrinsically critical. CIC critical point is achieved by setting model parameters to network-independent values. For CP, a known network-independent value is a good approximation of it. The critical point of SIS is approximated by considering the inverse of the largest eigenvalue of the adjacency matrix of the graph [17,44]. For SIR, the critical regime is approximated relying on the value of the largest eigenvalue of the nonbacktracking matrix of the graph $[45,46]$ (see Ref. [26] for further details).

The elementary rules at the basis of the various dynamical models are described in detail in the SM. Here, we briefly illustrate such rules for the sake of clarity. We remind that the underlying network is potentially directed, so that $A_{i j}=1$ indicates the existence of the connection $i \rightarrow j$. In the critical CIC, a randomly chosen node $i$ shares its state with all its neighbors, i.e., $\sigma_{j}(t+d t)=\sigma_{i}(t)$ for all $j$ such that $A_{i j}=1$, where $d t$ indicates the amount of time needed for the elementary reaction to occur. In the VOT model, a randomly chosen node $i$ inherits the state of one randomly chosen neighbor $j$, $\sigma_{i}(t+d t)=\sigma_{j}(t)$ with $A_{j i}=1$. In the IP, a randomly chosen node $i$ copies its own state on a randomly chosen neighbor $j$, $\sigma_{j}(t+d t)=\sigma_{i}(t)$ with $A_{i j}=1$. In the LD, a random edge $i \rightarrow j$ is first selected, then the state of node $j$ becomes identical to the one of node $i, \sigma_{j}(t+d t)=\sigma_{i}(t)$. In the CP, two possible events may happen: (i) a randomly chosen active node $i$ may recover, i.e., $\sigma_{i}(t)=1 \rightarrow \sigma_{i}(t+d t)=0$; (ii) a randomly chosen node $i$ spreads its activity on a randomly chosen neighbor $j$, i.e., $\sigma_{i}(t)=1$ and $A_{i j}=1$ causes $\sigma_{j}(t+$ $d t)=\sigma_{i}(t)$. Also in the SIS two possible events may occur: (i) a randomly chosen active node $i$ may recover, i.e., $\sigma_{i}(t)=$ $1 \rightarrow \sigma_{i}(t+d t)=0$; (ii) a random link $i \rightarrow j$ is chosen so that $\sigma_{i}(t)=1$ and $\sigma_{j}(t)=0$, then $\sigma_{j}(t)=0 \rightarrow \sigma_{j}(t+d t)=1$. Finally, the rules of the SIR are almost the same as those of SIS. The only difference is that the recovery event (i) leads to the change $\sigma_{i}(t)=1 \rightarrow \sigma_{i}(t+d t) \neq 0,1$, and nodes in the recovered state do not longer participate in the dynamics.

It is possible to classify the various models in three main classes of dynamical behavior. The first class is formed by the CP and IP, for which the expected number of spreading events in which the generic node $i$ influences its neighbors is a constant independent of the out-degree $k_{i}^{\text {out }}$. They differ from the CIC, VOT, LD, SIS, and SIR for which the expected number of spreading events in which node $i$ influences its neighbors is directly proportional to the out-degree $k_{i}^{\text {out }}$. As stressed above, the CIC, VOT, and LD are tuned to criticality in a way that is independent of the underlying network topology, thus they constitute a separate class from the one of the SIS and SIR models, whose critical regime is determined by the network topology.

We consider avalanches initiated by a single randomly chosen node, $j$, so that the initial configuration is $\sigma_{i}(0)=0$ for all $i \neq j$, and $\sigma_{j}(0)=1$. We follow the dynamics of each avalanche until the system reaches an absorbing configuration. We define the duration $T$ as the number of time steps needed 

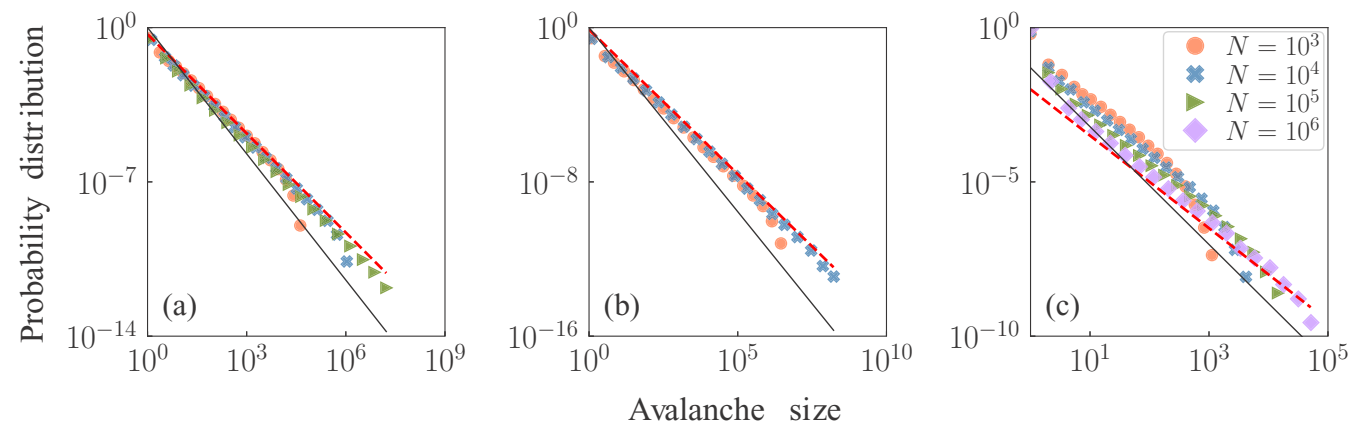

FIG. 2. Avalanche size in synthetic undirected scale-free networks. The degree exponent is $\gamma=2.1$. For clarity, we report results only for three dynamical models: (a) CIC, (b) IP, and (c) SIS. Results for all other models are in Ref. [26]. For each model and network, we measure, by means of numerical simulations, the probability distribution $P(S)$ of the total number of spreading events $S$ per avalanche. The dashed red line corresponds to MF exponents, i.e., Eq. (2); the full black line indicates anomalous BP scaling i.e., Eq. (4).

to reach an absorbing configuration. We also define the size $S$ as the number of elementary spreading events occurred during $T$. An elementary spreading event is the occurrence of an active node passing activation to other, not necessarily inactive, nodes.

All nodes may participate multiple times in an avalanche (i.e., they can be "reactivated") so that the network size $N$ is not an upper bound for $S$; the only exception to this rule is the SIR model where nodes can be activated only once. We focus on finite avalanches only, i.e., those that end up in absorbing configuration $\vec{\sigma}=\overrightarrow{0}$. In the CIC model, for instance, we exclude avalanches that end in $\vec{\sigma}=\overrightarrow{1}$, as they can be viewed as infinite avalanches. For the SIR model, we consider instead all avalanches.

We study, by means of extensive computational simulations, avalanche statistics for all the above-mentioned prototypical dynamical models on top of scale-free networks generated by one of two possible standard generative models, both of which produce uncorrelated random graphs with power-law degree distributions.

First, we consider undirected scale-free networks obtained via the uncorrelated configuration model [47] with degree distribution $P(k) \sim k^{-\gamma}$ with support $[4, \sqrt{N}]$. In our numerical analyses, we set $\gamma=2.1$ and vary the network size $N$. The choice $\gamma=2.1$ is expedient because it corresponds to a large gap between standard MF and ABP exponents, easing computational discrimination of scaling regimes. Results for $\gamma=2.5$ are reported in Ref. [26]. We generated a single graph instance of the model for every $N$, and used these graphs in all our analyses. We tested that choosing a particular instance of the graph model does not affect the statistics of avalanches. ${ }^{3}$ For every network and model, we simulated $10^{6}$ avalanches seeded at a randomly chosen single node and measured the corresponding avalanche size and duration distributions, i.e., $P(S)$ and $P(T)$, respectively.

The first major result of our analyses (see Fig. 2) is that — when deployed on undirected scale-free networks — all

\footnotetext{
${ }^{3}$ Please note that each network size corresponds indeed to a different instance of the network model. Observe that no significant variation among the various network instances is visible, if not due to finite-size effects.
}

activation models considered are characterized asymptotically (for large $S$ and large $T$ ) by standard MF exponents. This happens regardless of the fact that we have set $2<\gamma<3$, i.e., for networks for which a strict analogy with branching process would suggest anomalous exponents. An exhaustive report of the results of our analysis is shown in Ref. [26].

Second, we analyze directed scale-free networks constructed according to the model of Ref. [4]. This is a simple extension of the configuration model to generate directed networks, where node out-degrees $k^{\text {out }}$ are drawn from the distribution $P\left(k^{\text {out }}\right) \sim\left[k^{\text {out }}\right]^{-\gamma}$ for $k^{\text {out }} \in\left[4, k_{\max }^{\text {out }}\right]$ and $P\left(k^{\text {out }}\right)=$ 0 , otherwise ${ }^{4}$ (see Ref. [26]). We consider $\gamma=2.1$ as above (results for $\gamma=2.5$ are also reported in [26]) and set either $k_{\max }^{\text {out }}=\sqrt{N}$ or $k_{\max }^{\text {out }}=N-1$.

When $k_{\max }^{\text {out }}=\sqrt{N}$ our simulations show that all the activation processes are again in the MF universality class (see [26]) for all values of $\gamma$.

A more complicated scenario emerges when $k_{\max }^{\text {out }}=N-1$. In Fig. 3, we show the results only for few selected models. Results for all other models are reported in [26]. For IP and $\mathrm{CP}$, we still observe clear MF scaling, in all networks. In all other models, the distribution of the avalanche sizes displays a crossover from anomalous (for small sizes/durations) to $\mathrm{MF}$ exponents (for large sizes/durations). The crossover point increases with the network size, suggesting that only anomalous exponents should be present in the limit of asymptotically large networks. ${ }^{5}$

\footnotetext{
${ }^{4}$ In the generation of a graph instance, each node $i$ is connected to $k_{i}^{\text {out }}$ other nodes, chosen at random in the network, so that indegrees obey a Poissonian distribution with average value equal to the average out-degree.

${ }^{5}$ Let us stress that the observed scaling of $P(T)$ and power-law relation between $S$ and $T$ provide much less clear evidence of anomalous BP behavior even for the case $k_{\max }^{\text {out }}=N-1$ (see [26]). This issue is due to the finite size of the networks, and it is visible also in numerical results concerning pure BP with finite-size constraints [26]. Clearer observations of anomalous critical exponents for $P(T)$ and the power-law relation between $\langle S\rangle$ and $T$ can be obtained for $\gamma=2.5$; such a choice of the $\gamma$ value leads however to much less noticeable differences between anomalous and standard exponents for the distribution $P(S)$ [26]
} 

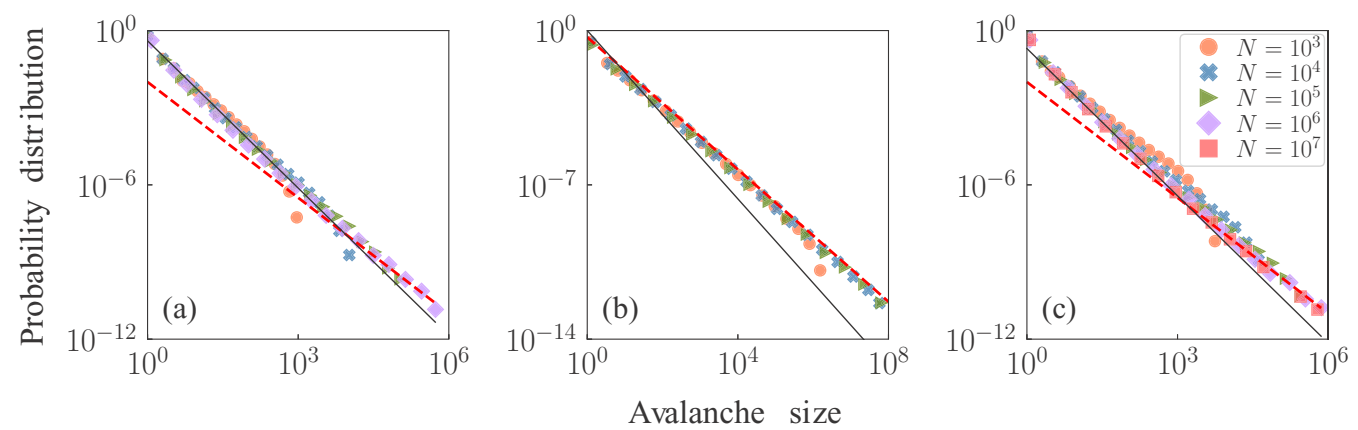

FIG. 3. Avalanche size in synthetic directed networks for three of the considered models: (a) CIC, (b) IP, and (c) SIS. The description of the figure panels is as in Fig. 2. The networks analyzed here are instances of the directed configuration model with out-degree exponent $\gamma=2.1$ and maximum out-degree $k_{\max }^{\text {out }}=N-1$.

In summary, our results provide strong support for MF exponents in all situations where a priori we expect standard BP behavior (i.e., finite second moment of the out-degree distribution). Settings for which one could predict a priori anomalous BP scaling (i.e., for scale-free networks with $2<$ $\gamma<3$ ) generate results that are much less cleancut. Anomalous exponents can at most be observed only in the regime of small avalanches for the distribution of the avalanche size. Strong deviations from the predicted anomalous power-law scalings are observed otherwise. An important role for the observation of anomalous exponents is played by the upper bound of the out-degree distribution. The magnitude of the largest degree, and in general, the frequency of high degree nodes determine the quantity of superspreaders and it is therefore a crucial quantity to consider in diffusion models. The upper bound determines how fast the second moment of the distribution diverges as the network size is increased. On unweighted networks, maximal divergence is obtained for $k_{\max }=N-1$, corresponding to the setting where anomalous scaling can be best appreciated. Slow divergence, as for the case $k_{\max }^{\text {out }}=\sqrt{N}$, makes it difficult to observe anomalous behavior, at least for the size of the networks that we are considering in this work.

\section{ANALYTICAL APPROACH}

Two alternative types of approaches are frequently used to study avalanches in networks. The first one is, as discussed above, a branching process approximation for cascades of a small size at the beginning of the process. The second one is an approximation by a dynamical system once the avalanche spreads to a significant fraction of the underlying network [48]. The mathematical approach we develop in what follows belongs to this second group.

Depending on their specific features, all dynamical models under consideration can be grouped into three classes described by different types of mathematical equations. These groups are (i) IP and CP; (ii) CIC, VOT, and LD; (iii) SIS and SIR.

The first group is trivially described by dynamical processes that are insensitive to the out-degree sequence of the underlying network. In other words, anomalous propagation events in which a single active node propagates activity to an arbitrarily large number of nearest neighbors are simply not allowed by the dynamics. Henceforth, anomalous type of scaling is not expected to appear, even at the level of a naive mapping onto a branching process. Thus, IP and $\mathrm{CP}$ avalanches are expected to be always characterized by standard MF exponents (see Ref. [26]), in perfect agreement with our computational results.

Models in the other two classes have instead a much less trivial behaviour. We consider the CIC and SIS models as representative of each of these two classes and derive a mathematical approach for each. The full development of the theory (and extension to the other dynamical models) is presented in Ref. [26]; here, we sketch the main results and the main insights derived from them.

Our analytical approach is based on two successive approximations. The first one is the so-called individual-based meanfield approximation (IBMFA) (see Pastor-Satorras et al. [2] for a review). This analysis starts by describing the evolution of the average value of the state of an individual node in the network, where the average is taken over many realizations of the dynamical process. The approximation consists in neglecting dynamical correlations among variables, so that every node feels only the influence of the average behavior of each of its neighbors. We use the IBMFA for determining how and when the system reaches its long-term dynamical regime. The second approximation consists in deriving a Langevin equation for the overall network activity, written as the sum of the activity variables of all nodes [49]. From this approximation, it is possible to derive the statistics of long-term avalanches based on the equivalence between the resulting Langevin equations and Eq. (3), derived in Ref. [31] to describe the standard branching process and related processes.

Let us first present the derivation of the main results for CIC critical dynamics, in which the only possible change in the state of a node consists in copying the state of a nearest neighbor. In the IBMFA, we focus our attention on the deterministic node variable $s_{i}(t):=\left\langle\sigma_{i}(t)\right\rangle$, defined as the value of the stochastic variable $\sigma_{i}(t)$, averaged over the realizations of the dynamical process at time $t$. As we explicitly derive in Ref. [26], critical CIC dynamics is described by the IBMFA equation

$$
\frac{d \vec{s}(t)}{d t}=-L^{T} \vec{s}(t)
$$


Here, $\overrightarrow{s(t)}=\left[s_{1}(t), s_{2}(t), \ldots, s_{N}(t)\right]^{T}$ is the vector describing the average state of the nodes of the network at time $t$. $L=K^{i n}-A$ is the (directed) graph Laplacian of the network, with $K^{\text {in }}$ the diagonal matrix whose non-null elements are equal to the in-degree of the nodes, and $A$ is the graph adjacency matrix [50]. In essence, under the IBMFA, the critical CIC coincides with a purely diffusive process. The properties of the solutions of Eq. (5) for arbitrary graphs are described in Refs. [51,52]; we briefly summarize them here. If the underlying network is composed of a single strongly connected component, then, the long-term behavior is such that $\lim _{t \rightarrow \infty} s_{i}(t)=s^{*}$, for all nodes $i$. Because the asymptotic limit of the individual variables $s_{i}$ does not depend on $i$, $s^{*}$ coincides with the asymptotic value of density, $r^{*}$. The latter is given by the norm of the vector $s(t)$ at large times, i.e., $r^{*}=\left\|\left[\left(\vec{v}_{1}^{(l)}\right)^{T} \cdot \vec{s}(t=0)\right] \cdot \vec{v}_{1}^{(r)}\right\|$, where $\vec{v}_{1}^{(l)}$ and $\vec{v}_{1}^{(r)}$ are the left and right eigenvectors of $L^{T}$, respectively. These eigenvectors correspond to the smallest eigenvalue $v_{1}=0$ of the graph Laplacian. The long-term regime is reached exponentially fast. However, depending on the type of network, we have different diffusion behaviors. For undirected networks, $\vec{v}_{1}^{(l)}=\vec{v}_{1}^{(r)}=\overrightarrow{1} / \sqrt{N}$; further, the density of active nodes $r(t):=1 / N \sum_{i} s_{i}(t)$ is such that $r(t)=r(0)=1 / N$ for all $t$. The typical timescale is $t^{*}=1 / \nu_{2}$, with $\nu_{2}$ the second smallest eigenvalue of $L$ (see [26]).

On the other hand, if the network is directed, $\vec{v}_{1}^{(r)}=\overrightarrow{1} / \sqrt{N}$, but $\vec{v}_{1}^{(l)} \neq \vec{v}_{1}^{(r)}$. This means that, also in this case the vector $\vec{s}$ has identical components for $t \rightarrow \infty$. However, $r(t)$ may increase or decrease depending on the initial condition, so that the steady-state value $r^{*}$ of the density is sensitive to the initial choice of the seed node. ${ }^{6}$ Further, in this case, we cannot longer apply the spectral theorem to the corresponding non-symmetric matrix so that the relaxation to the steady-state cannot be easily written in terms of the Laplacian eigenvalues.

To determine the statistical properties of avalanches with duration $T \gg t^{*}$, i.e., asymptotically, we now go back to the stochastic description of the full dynamical system. We take advantage of the previous finding obtained under the IBMFA, and assume that $\rho(t):=1 / N \sum_{i} \sigma_{i}(t)$ is a quantity that fluctuates around its average value $\langle\rho\rangle=r^{*}$. Essentially, we make the hypothesis that the system has reached a stationary state where the number of active nodes is constant on average, but still subjected to demographic fluctuations. In analogy with Ref. [49], we refer to this assumption as the adiabatic approximation. Thus, the dynamics of long-term avalanches in CIC critical dynamics turns out to obey the following Langevin equation (see Ref. [26])

$$
\frac{d \rho(t)}{d t}=\sqrt{2\left\langle k^{i n}\right\rangle / N} \sqrt{\rho(t)[1-\rho(t)]} \xi(t),
$$

where $\xi(t)$ is a zero-mean Gaussian white noise, and $\left\langle k^{i n}\right\rangle$ is the average in-degree of the network. The dependence on $\rho$ of the diffusion coefficient imposes the absence of fluctuations for both $\rho=0$ and $\rho=1$, corresponding to the two existing

\footnotetext{
${ }^{6}$ For instance, in [26] we show that for the directed configuration model, the $i$-th component of such a vector is proportional to the out-degree of node $i$, i.e., $v_{1, i}^{(l)} \sim k_{i}^{\text {out }}$.
}

absorbing states. Except for higher-order terms, Eq. (6) has the generic form of the representative Langevin equation, Eq. (3), for avalanches in the class of standard MF branching processes [31]. This implies that long-term avalanches in critical CIC dynamics obey power-law distributions with MF critical exponents, i.e., Eqs. (2).

We now briefly illustrate the analytical approach for SIS critical dynamics. We basically repeat the same steps described above for critical CIC dynamics. The IBMFA equation reads as

$$
\frac{d \vec{s}(t)}{d t}=\left(A^{T}-I\right) \vec{s}(t),
$$

where $I$ is the identity matrix [44]. The solution of the IBMFA equation is a vector whose components are proportional to those of the principal right eigenvector $\vec{w}_{N}^{(r)}$ of the matrix $A^{T}$ [44]; convergence to the asymptotic solution is exponentially fast. The asymptotic value of density of active nodes is given by $\left.r^{*}=\|\left(\vec{w}_{N}^{(l)}\right)^{T} \cdot \vec{s}(t=0)\right] \vec{w}_{N}^{(r)} \|$, with $\vec{w}_{N}^{(l)}$ and $\vec{w}_{N}^{(r)}$ principal left and right eigenvector of the matrix $A^{T}$ respectively. ${ }^{7}$ If the network is undirected, the timescale of the exponential relaxation to the steady-state density is given by $t^{*}=\omega_{N} /\left(\omega_{N}-\omega_{N-1}\right)$, with $\omega_{N}$ largest eigenvalue of $A$, and $\omega_{N-1}$ second largest eigenvalue of $A$ (see [26]). If the network is directed, $t^{*}$ is not directly quantifiable in terms of the eigenvalues of the matrix $A$.

For $t \gg t^{*}$, the system has reached its long-term dynamical regime. The statistics of long avalanches is described by the Langevin equation

$$
\frac{d \rho(t)}{d t}=\sqrt{2\left\langle w_{N}^{(r)}\right\rangle / N} \sqrt{\rho(t)} \xi(t) .
$$

where $\left\langle w_{N}^{(r)}\right\rangle$ is the average value of the components of the principal right eigenvector of the matrix $A^{T}$ (see Ref. [26]). Eq. (8) has the same form as those considered by di Santo et al. [31], valid for avalanche models that are equivalent to standard BP processes. This tells us that long-term avalanches in critical SIS dynamics obey power-law distributions with MF critical exponents, i.e., Eq. (2).

In summary, the above analytical approach tells us that sufficiently long (large) avalanches in critical CIC and SIS dynamics should follow a standard MF scaling. This conclusion, in principle is true for any network. However, an avalanche is sufficiently long to obey standard BP statistics only if its duration is much longer than the typical timescale that can be deduced from the IBMFA of the process happening on the network. The magnitude of such timescale depends exclusively on the topology of the network, by means of either the Laplacian or the adjacency matrix of the graph. Undirected networks with sufficiently short average distance, for instance, have a relatively small diffusion timescale. There are, however, network topologies where diffusion may be particularly slow to reach its stationary state. Examples are low-dimensional lattices, and networks with long loops. In

\footnotetext{
${ }^{7}$ In uncorrelated random network models, the components of the vector $\vec{w}_{N}^{(l)}$ are proportional to the node out-degrees, i.e., $w_{1, i}^{(l)}$ $\sim k_{i}^{\text {out }}$ (see [26]). For undirected configuration models, the previous statement is valid only when the degree exponent $\gamma<5 / 2$.
} 

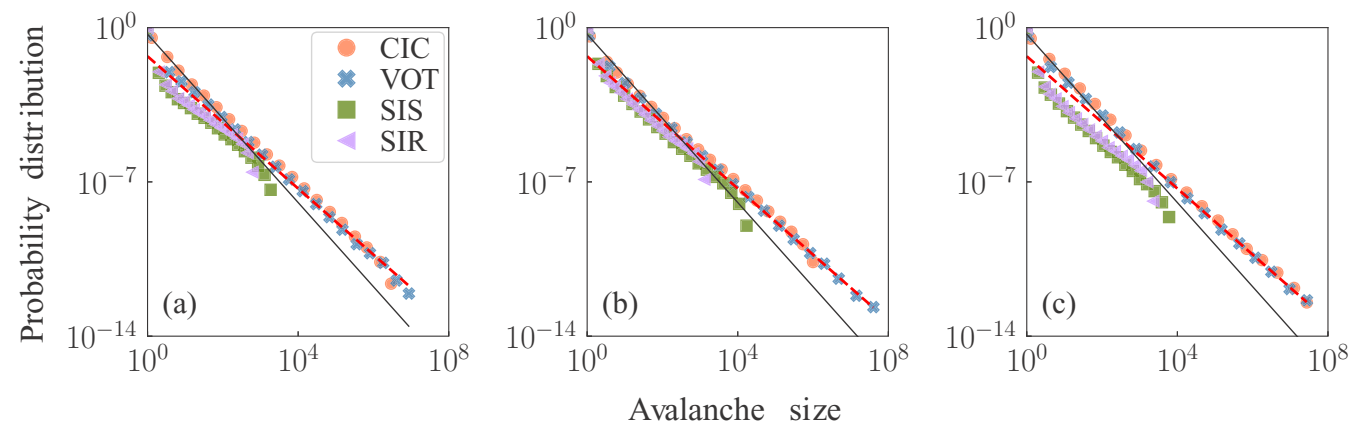

FIG. 4. Avalanche size in real-world networks. We consider the following networks: (a) undirected graph representing a snapshot of the Internet at the Autonomous system level [53]; (b) directed Twitter network of the Spanish 15M movement [54]; (c) directed graph representing a portion of the YouTube social network [55]. Different symbols and colors refer to different avalanche dynamical models. The red dashed line represent standard BP critical exponents, while the full black line indicates the power-law decay expected for anomalous BP. Note that the out-degree distributions of these networks are all well modeled by power laws with decay exponent $\gamma=2.1$ (see Ref. [26]).

these cases, the vast majority of observed avalanches may never be long enough as to be describable by the long-term statistics. We do not have analytical arguments to determine the statistical properties of avalanches in the short-term dynamical regime, but, in principle, one expects that the effective mapping into an ABP should work (for scale-free networks with $2<\gamma<3$ ). Our numerical results seem to indicate that anomalous BP scaling is possible as long as the underlying networks are directed and have power-law out-degree distributions. The cutoff of the out-degree distribution seems also to play an important role for the possible observation of anomalous scaling, at least for the network sizes that we were able to consider in our analysis.

\section{REAL NETWORKS}

All considerations, numerical and theoretical, made for synthetic graphs are valid also for real-world networks. In Fig. 4, we summarize the results of numerical simulations performed on three large-scale networks. Additional results are provided in Ref. [26]. A pre-asymptotic regime with anomalous scaling for sufficiently small avalanches is seen for example in the YouTube direct social network [Fig. 4(c)]. The distributions for large avalanches are instead very well described by MF critical exponents in all cases. The crossover point may be interpreted as the typical scale that distinguishes local from global avalanches, and it could be employed as a quantitative criterion to tell whether an avalanche is "viral" or not.

\section{v. CONCLUSIONS AND DISCUSSION}

In conclusion, we found that any minimal deviation from the assumptions underlying the mapping into an anomalous branching process brings the system back to the realm of standard MF and its associated superuniversal exponents, so that anomalous exponents are exceedingly difficult to observe. Our results suggest this statement to be true for seven wellknown avalanche dynamical models, but we believe that it can be extended to many other spreading processes taking place on networks. Our results are valid for avalanche dynamical models with asynchronous updating rules. We do not exclude that models with synchronous updates may exhibit different statistical properties, with anomalous exponents emerging even in settings less peculiar than those identified here for asynchronous dynamical models [56].

Why is numerical evidence of anomalous scaling so weak, even in the cases when intuition suggests that the dynamical avalanche model could be well mapped to an anomalous branching process? Clearly, if the process is occurring on a directed tree with power-law out-degree distribution, then anomalous scaling occurs. However, avalanche models in more complex networks do not necessarily satisfy such strict conditions. There are many possible ways in which the assumptions of the mapping to an anomalous branching process can be violated.

First, both in directed and undirected networks avalanches do not necessarily proceed in a fully feedforward way; already active nodes can be found by a branch of an unfolding avalanche thus breaking the equivalence with a pure branching process. In other words, feedforward loops may exist, meaning that a given node can be reached from a unique seed by following different paths. This is particularly relevant in undirected networks, where activity can attempt to go backwards after any propagation event, following a reversible link. This type of interference reduces the effective number of independent offspring, breaking the BP analogy.

Second, networks in simulations are finite, implying that a finite maximum degree exists, therefore the out-degree variance takes a finite value; this implies that there should be crossovers to the standard exponents for sufficiently large avalanche sizes and durations.

Last but not least, some types of dynamics, even if taking place on top of scale-free networks, do not really involve all neighbors of a single node--as for example in the CP and IP processes - and, thus, have an effective offspring distribution narrowly distributed, implying the emergence of standard MF exponents.

Numerous real-world systems have been investigated in terms of avalanche statistics. Prototypical examples include natural systems, such as neuronal networks [57], $\gamma$-ray bursts [58] and earthquakes [59], as well as socio-technical systems, such as power networks [60] and online social media [61-64]. 
Among them, some systems display critical avalanche statistics consistent with the MF universality class [57]. However, there are many other systems showing avalanche statistical properties that are not consistent with the MF universality class. Examples can be found especially in the literature studying information avalanches in online social media where measured exponents for the power-law distribution of avalanche size range from $\tau \simeq 4$ [61], to $\tau \simeq 2.3$ [63] and $\tau \simeq 2$ [62].

Our analytical and numerical evidence supports the existence of an extremely robust universality class at the interface between the absorbing and the active phases of many popular models of avalanche dynamics. Such a universality class can be broken only at the expense of making specific assumptions on the shape of the network underlying the spreading model. We believe that it is imperative to understand why there exist real systems that do not conform to such a class, and what alternative hypotheses need to be made to account for their behavior. In other words, a complete analytical theoryextending the approach presented here-and accounting for all types of networks still needs to be constructed.
As a final note, let us stress that our results reveal that constructing a dynamical model characterized by $\mathrm{MF}$ avalanche exponents is not a hard task. Consequently, having a model that generates avalanche distributions with MF exponents-being these in agreement with some experimental observation-does not constitute a sufficient evidence that the model is actually a sound one. Other dynamical aspects should be also used to validate the model.

\section{ACKNOWLEDGMENTS}

F.R. and D.N. acknowledge support from the National Science Foundation (CMMI-1552487). F.R. acknowledges support from the US Army Research Office (W911NF16-1-0104). M.A.M. acknowledges the Spanish Ministry and Agencia Estatal de investigación (AEI) through grant FIS2017-84256-P (European Regional Development Fund), as well as the Consejería de Conocimiento, Investigación y Universidad, Junta de Andalucía and European Regional Development Fund (ERDF), ref. A-FQM-175-UGR18 and SOMM17/6105/UGR for financial support.
[1] C. Castellano, S. Fortunato, and V. Loreto, Statistical physics of social dynamics, Rev. Mod. Phys. 81, 591 (2009).

[2] R. Pastor-Satorras, C. Castellano, P. Van Mieghem, and A. Vespignani, Epidemic process in complex networks, Rev. Mod. Phys. 87, 925 (2015).

[3] O. A. Pinto and M. A. Muñoz, Quasi-neutral theory of epidemic outbreaks, PLoS One 6, e21946 (2011).

[4] J. P. Gleeson, J. A. Ward, K. P. O'Sullivan, and W. T. Lee, Competition-Induced Criticality in a Model of Meme Popularity, Phys. Rev. Lett. 112, 048701 (2014).

[5] D. Notarmuzi and C. Castellano, Analytical study of qualitybiased competition dynamics for memes in social media, Europhys. Lett. 122, 28002 (2018).

[6] T. M. Liggett, Stochastic models of interacting systems, Ann. Probab. 25, 1 (1997).

[7] P. L. Krapivsky, Kinetics of monomer-monomer surface catalytic reactions, Phys. Rev. A 45, 1067 (1992).

[8] C. Castellano, Effect of network topology on the ordering dynamics of voter models, in AIP Conference Proceedings (AIP, 2005), Vol. 779, pp. 114-120.

[9] V. Sood, T. Antal, and S. Redner, Voter models on heterogeneous networks, Phys. Rev. E 77, 041121 (2008).

[10] R. Durrett, Oriented percolation in two dimensions, Ann. Probab. 12, 999 (1984).

[11] J.-P. Onnela and F. Reed-Tsochas, Spontaneous emergence of social influence in online systems, Proc. Natl. Acad. Sci. USA 107, 18375 (2010).

[12] K. Lerman, R. Ghosh, and T. Surachawala, Social contagion: An empirical study of information spread on digg and twitter follower graphs, arXiv:1202.3162.

[13] M. A. Muñoz, Colloquium: Criticality and dynamical scaling in living systems, Rev. Mod. Phys. 90, 031001 (2018).

[14] M. E. J. Newman, S. H. Strogatz, and D. J. Watts, Random graphs with arbitrary degree distributions and their applications, Phys. Rev. E 64, 026118 (2001).
[15] R. Cohen, D. ben-Avraham, and S. Havlin, Percolation critical exponents in scale-free networks, Phys. Rev. E 66, 036113 (2002).

[16] S. N. Dorogovtsev, A. V. Goltsev, and J. F. F. Mendes, Critical phenomena in complex networks, Rev. Mod. Phys. 80, 1275 (2008).

[17] D. B. Larremore, M. Y. Carpenter, E. Ott, and J. G. Restrepo, Statistical properties of avalanches in networks, Phys. Rev. E 85, 066131 (2012).

[18] H. W. Watson and F. Galton, On the probability of the extinction of families, J. Anthropol. Inst. (Great Britain and Ireland) 4, 138 (1875).

[19] T. E. Harris, The Theory of Branching Processes (Dover, New York, 1989).

[20] W. Feller, Two singular diffusion problems, Ann. Math. 54, 173 (1951).

[21] T. M. Liggett, Interacting Particle Systems, Classics in Mathematics (Springer, New York, 2004).

[22] L. P. Kadanoff, S. R. Nagel, L. Wu, and S.-M. Zhou, Scaling and universality in avalanches, Phys. Rev. A 39, 6524 (1989).

[23] M. A. Muñoz, R. Dickman, A. Vespignani, and S. Zapperi, Avalanche and spreading exponents in systems with absorbing states, Phys. Rev. E 59, 6175 (1999).

[24] J. P. Sethna, K. A. Dahmen, and C. R. Myers, Crackling noise, Nature (London) 410, 242 (2001).

[25] A. Baldassarri, F. Colaiori, and C. Castellano, Average Shape of a Fluctuation: Universality in Excursions of Stochastic Processes, Phys. Rev. Lett. 90, 060601 (2003).

[26] See Supplemental Material at http://link.aps.org/supplemental/ 10.1103/PhysRevResearch.2.033171 for a detailed description of the various dynamical models and network topologies used in the analysis, additional numerical results, and full development of the theoretical approach. 
[27] M. A. Muñoz, G. Grinstein, and Y. Tu, Survival probability and field theory in systems with absorbing states, Phys. Rev. E 56, 5101 (1997).

[28] H. K. Janssen, Survival and percolation probabilities in the field theory of growth models, J. Phys. Cond. Matter 17, S1973 (2005).

[29] S. Redner, A Guide to First-Passage Processes (Cambridge University Press, Cambridge, 2001).

[30] M. Henkel, H. Hinrichsen, and S. Lübeck, Non-Equilibrium Phase Transitions: Absorbing Phase Transitions, Theoretical and Mathematical Physics (Springer London, Berlin, 2008).

[31] S. di Santo, P. Villegas, R. Burioni, and M. A. Muñoz, Simple unified view of branching process statistics: Random walks in balanced logarithmic potentials, Phys. Rev. E 95, 032115 (2017).

[32] M. Plischke and B. Bergersen, Equilibrium Statistical Physics (World Scientific, Singapore, 2006).

[33] S. Zapperi, K. B. Lauritsen, and H. E. Stanley, Self-Organized Branching Processes: Mean-Field Theory for Avalanches, Phys. Rev. Lett. 75, 4071 (1995).

[34] R. Otter, The multiplicative process, Ann. Math. Stat. 20, 206 (1949).

[35] J. Marro and R. Dickman, Nonequilibrium Phase Transition in Lattice Models (Cambridge University Press, Cambridge, 1999).

[36] K.-I. Goh, D.-S. Lee, B. Kahng, and D. Kim, Sandpile on ScaleFree Networks, Phys. Rev. Lett. 91, 148701 (2003).

[37] A. Saichev, A. Helmstetter, and D. Sornette, Power-law distributions of offspring and generation numbers in branching models of earthquake triggering, Pure Appl. Geophys. 162, 1113 (2005).

[38] D. Sornette, Critical Phenomena in Natural Sciences: Chaos, Fractals, Selforganization and Disorder: Concepts and Tools, Springer Series in Synergetics (Springer, 2006).

[39] A.-L. Barabási and R. Albert, Emergence of scaling in random networks, Science 286, 509 (1999).

[40] I. Voitalov, P. van der Hoorn, R. van der Hofstad, and D. Krioukov, Scale-free networks well done, Phys. Rev. Research 1, 033034 (2019).

[41] P. Grassberger, On the critical behavior of the general epidemic process and dynamical percolation, Math. Biosci. 63, 157 (1983).

[42] R. Cohen, K. Erez, D. ben-Avraham, and S. Havlin, Resilience of the Internet to Random Breakdowns, Phys. Rev. Lett. 85, 4626 (2000).

[43] F. Radicchi and C. Castellano, Breaking of the site-bond percolation universality in networks, Nat. Commun. 6, 10196 (2015).

[44] A. V. Goltsev, S. N. Dorogovtsev, J. G. Oliveira, and J. F. F. Mendes, Localization and Spreading of Diseases in Complex Networks, Phys. Rev. Lett. 109, 128702 (2012).

[45] B. Karrer, M. E. J Newman, and L. Zdeborová, Percolation on Sparse Networks, Phys. Rev. Lett. 113, 208702 (2014).

[46] F. Radicchi, Predicting percolation thresholds in networks, Phys. Rev. E 91, 010801(R) (2015).
[47] M. Catanzaro, M. Boguñá, and R. Pastor-Satorras, Generation of uncorrelated random scale-free networks, Phys. Rev. E 71, 027103 (2005).

[48] R. Rastegar and A. Roitershtein, Avalanches in an excitable network, arXiv:1904.01220.

[49] M. Boguñá, C. Castellano, and R. Pastor-Satorras, Langevin approach for the dynamics of the contact process on annealed scale-free networks, Phys. Rev. E 79, 036110 (2009).

[50] J. S. Caughman and J. J. P. Veerman, Kernels of directed graph laplacians, Electron. J. Comb. 13, 39 (2006).

[51] N. Masuda, M. A. Porter, and R. Lambiotte, Random walks and diffusion on networks, Phys. Rep. 716, 1 (2017).

[52] J. J. P. Veerman and E. Kummel, Diffusion and consensus on weakly connected directed graphs, arXiv:1807.09846.

[53] J. Leskovec, J. Kleinberg, and C. Faloutsos, Graphs over time: Densification laws, shrinking diameters and possible explanations, in Proceedings of the Eleventh ACM SIGKDD International Conference on Knowledge Discovery in Data Mining (ACM, 2005), pp. 177-187.

[54] J. Borge-Holthoefer, A. Rivero, I. García, E. Cauhé, A. Ferrer, D. Ferrer, D. Francos, D. Iñiguez, M.P. Pérez, G. Ruiz et al., Structural and dynamical patterns on online social networks: The spanish may 15th movement as a case study, PLoS One 6, e23883 (2011).

[55] A. Mislove, M. Marcon, K.P. Gummadi, P. Druschel, and B. Bhattacharjee, Measurement and Analysis of Online Social Networks, in Proceedings of the 5th ACM/Usenix Internet Measurement Conference (IMC'07) ( San Diego, CA, 2007).

[56] J. Zierenberg, J. Wilting, V. Priesemann, and A. Levina, Description of spreading dynamics by microscopic network models and macroscopic branching processes can differ due to coalescence, Phys. Rev. E 101, 022301 (2020).

[57] J. M. Beggs and D. Plenz, Neuronal avalanches in neocortical circuits, J. Neurosci. 23, 11167 (2003).

[58] F. Y. Wang and Z. G. Dai, Self-organized criticality in x-ray flares of gamma-ray-burst afterglows, Nat. Phys. 9, 465 (2013).

[59] P. Bak, K. Christensen, L. Danon, and T. Scanlon, Unified Scaling Law for Earthquakes, Phys. Rev. Lett. 88, 178501 (2002).

[60] R. Kinney, P. Crucitti, R. Albert, and V. Latora, Modeling cascading failures in the north american power grid, EPJB 46, 101 (2005).

[61] R. Nishi, T. Takaguchi, K. Oka, T. Maehara, M. Toyoda, K. Kawarabayashi, and N. Masuda, Reply trees in Twitter: Data analysis and branching process models, Soc. Netw. Anal. Min. 6, 26 (2016).

[62] X. Qiu, D. F. M. Oliveira, A. S. Shirazi, A. Flammini, and F. Menczer, Limited individual attention and online virality of low-quality information, Nat. Hum. Beh. 1, 0132 (2017).

[63] K. Wegrzycki, P. Sankowski, A. Pacuk, and P. Wygocki, Why do cascade sizes follow a power-law? Proceedings of the 26th International Conference on World Wide Web (ACM, Perth, Australia, 2017).

[64] K. Lerman and R. Ghosh, Information contagion: An empirical study of the spread of news on digg and twitter social networks, in Fourth International AAAI Conference on Weblogs and Social Media (AAAI Press, Washington, D.C., 2010). 